\title{
An Approach for ECG Feature Extraction and Classification of Cardiac Abnormalities
}

\section{Sumathi S*}

Mahendra Engineering College, Namakkal, Tamilnadu, India

\begin{abstract}
This inquires about article presents a unused approach to the Programmed location and classification of electrocardiogram (ECG) signals is of tremendous significance for determination of cardiac anomalies. A strategy is proposed here to classify distinctive cardiac variations from the norm like Ventricular Arrythmias, Myocardial infarction, Myocardial hypertrophy and Valvular heart malady. Support Vector Machine (SVM) has been utilized to classify the designs inborn in the highlights extricated through Continuous Wavelet Transform (CWT) of distinctive ECG signals. CWT permits a time space flag to be changed into time-frequency space such that recurrence characteristics and the area of specific highlights in a time arrangement may be highlighted at the same time. Hence it permits precise extraction of highlight from non-stationary signals like ECG. At that point the support vector machine (SVM) with Gaussian part is utilized to classify diverse ECG heart cadence. In the display work, SVM in relapse mode has been effectively applied.
\end{abstract}

Keywords: ECG characterization; Wavelet transform; Feature mapping; FIR filter; Support vector

\section{Introduction}

The electrocardiogram (ECG) is routinely used in clinical practice, which describes the electrical activity of the heart. In physical checkups at hospitals, physicians record the ECG after the patient has exercised to check his/her cardiac condition. The Holter ECG device is used most frequently for recording the ECG. Physicians apply the device to a patient when they need to monitor his/her ECG to find the few abnormal cycles in the ECG throughout the day. Physicians then interpret the shapes of those waves and complexes. They calculate parameters to determine whether the ECG shows signs of cardiac disease or not. The parameters are the height and the interval of each wave, such as RR interval, PP interval, QT interval, and ST segment. Recognition of the fiducial points and calculations of the parameters is a tedious routine for the physician. Therefore, there is an urgent need for an automatic ECG recognition system to reduce the burden of interpreting the ECG. Various studies have been done for classification of various cardiac arrhythmias [1-4]. In this paper, we propose the combination of wavelet transform and AR model as the feature extraction method, then use the SVM to classify the ECG heartbeat. The proposed approach is validated in the MIT-BIH Arrhythmia Database [5]. Figure 1 shows an ideal ECG wave form. Various studies have been done for classification of various cardiac arrhythmias [1-4]. In this paper, we propose the combination of wavelet transform and AR model as the feature extraction method, then use the SVM to classify the ECG heartbeat. The proposed approach is validated in the MIT-BIH Arrhythmia Database [5-10]. Figure 1 shows an ideal ECG wave form.

\section{Filtering of ECG signal}

ECG signals of the over said illnesses along with the ECG flag of sound people have been collected from the PTB Demonstrative ECG Database www.physionet.org. The ECG signals have been recorded for 2 minutes and 30 seconds, with a testing recurrence of $1 \mathrm{kHz}$ for the 12 ECG leads. ECG signals are continuously influenced by commotion, pattern float and artifacts. Subsequently it is troublesome to utilize them in the crude shape for classification of malady. Sifting of the ECG signals are hence vital. In arrange to diminish exceptionally moo recurrence as well as the tall recurrence commotions, an FIR band

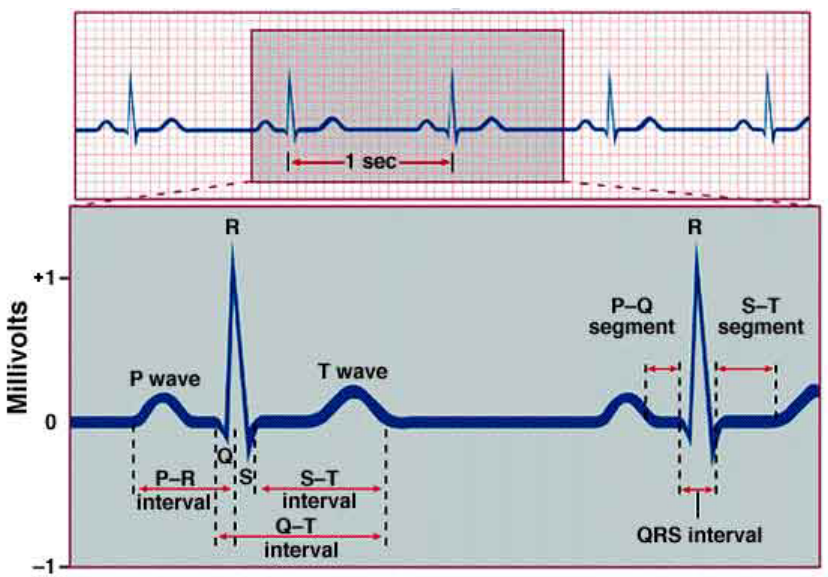

Figure 1: Ideal ECG wave form.

pass channel with the pass band $0.1-\mathrm{Hz}$ to $90 \mathrm{~Hz}$ has been utilized. The fundamental advantage of utilizing an FIR channel is that it minimizes waveform twisting and it moreover has about straight stage relationship. The greatness and stage reactions of the proposed FIR channel are appeared in Figures 2 and 3 appears the unique ECG flag taken from the PTB database and the yield flag of the said FIR channel.

\section{Wavelet transform}

The ECG signals are considered as representative signals of cardiac physiology, which are helpful in diagnosing cardiac disorders. The

*Corresponding author: Sumathi S, Professor, Department of Electrical and Electronics Engineering, Mahendra Engineering College (Autonomous), Namakkal, Tamilnadu, 637001, India, Tel: +91 9443745077; E-mail: drssumathiphd@gmail.com

Received February 05, 2018; Accepted February 21, 2018; Published February 27, 2018

Citation: Sumathi S (2018) An Approach for ECG Feature Extraction and Classification of Cardiac Abnormalities. Cardiovasc Pharm Open Access 6: 234. doi: 10.4172/23296607.1000234

Copyright: (C) 2018 Sumathi S. This is an open-access article distributed under the terms of the Creative Commons Attribution License, which permits unrestricted use, distribution, and reproduction in any medium, provided the original author and source are credited. 

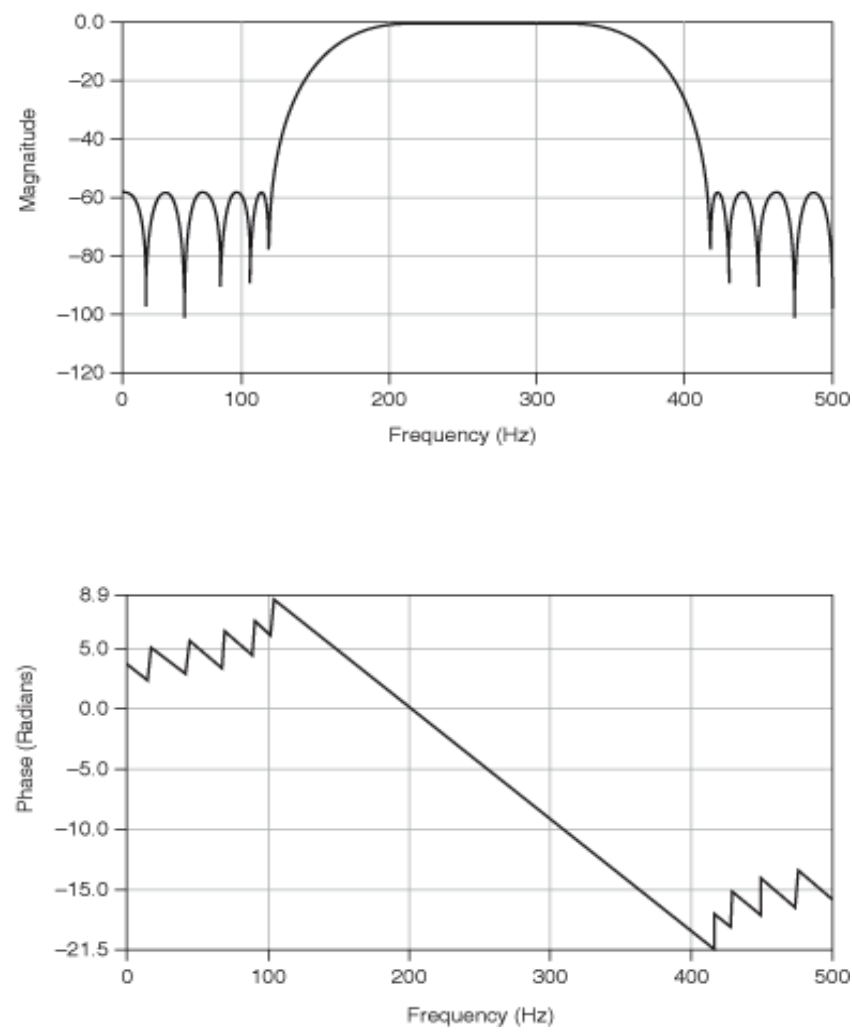

Figure 2: Magnitude and phase responses of the proposed FIR filter.

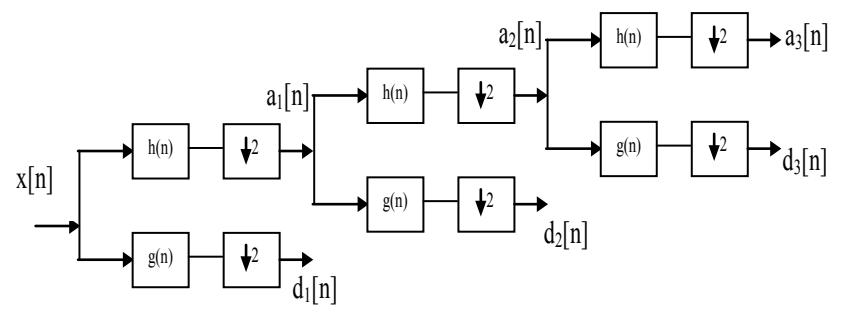

Figure 3: Three level wavelet decomposition tree.

absolute way to display this information is to perform spectral analysis. The Wavelet Transform (WT) gives very general techniques, which can be implemented to many tasks in signal processing. The ECG signal, consisting of many characteristic points, can be compressed into a few points. These points characterize the behaviour of the ECG signal. This feature of using a lesser number of parameters to represent the ECG signal is particularly important for recognition and diagnostic functions. The WT can be thought of as an extension of the classic Fourier transform, but instead of working on a single scale (time or frequency), it works on a multiple-scale basis. This multiple-scale feature of the WT allows the decomposition of a signal into a number of scales, every scale representing a particular coarseness of the signal under this study. The procedure of multiresolution decomposition of a signal $x[n]$ is schematically shown in Figure 4. Every stage of this scheme consists of two digital filters and two down samplers by 2 . The initial filter, the discrete mother wavelet is $g[\mathrm{n}]$, high pass in nature, and the second, $h[\mathrm{n}]$ is it's reflect version, lowpass in nature. The down sampled outputs of first high pass and low-pass filters give the detail, $d 1$ and the approximation, $a 1$, respectively. The first approximation, $a 1$ is more decomposed and this process is continued as shown in Figures 2 and 3 [11,12].

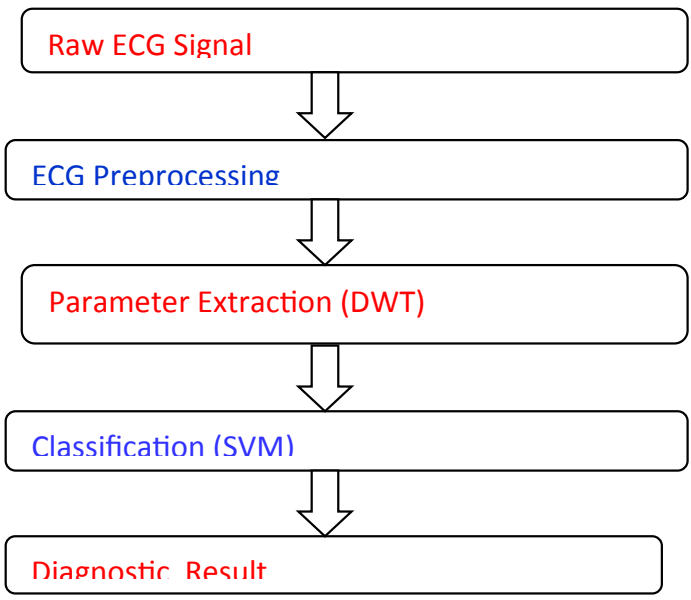

Figure 4: Proposed method for ECG beat classification.

\section{ECG database}

Physio Bank database is a large and growing archive of wellcharacterized digital recordings of physiological signals and related data for use by the biomedical research community. The databases of MIT-BIH Arrhythmia Database, MIT-BIH Atrial Arrythmia Database and Malignant Ventricular Arrhythmia Database were used for the analysis.

\section{THEORY of SVM}

The SVM technique was originally proposed essentially for classification problems of two classes but was found to be useful to deal with non-linearly separable cases too. Given a set of points which belong to either of two classes, a linear SVM finds the hyperplane leaving the largest possible fraction of points of the same class on the same side, while maximizing the distance of either class from the hyperplane [5-10].

$$
\begin{aligned}
& u(x)=\sum_{i=1}^{N} \alpha_{i} y_{i} K\left(x_{i}, x\right)+b \\
& F_{i}=\sum_{j=1}^{N} \alpha_{j} y_{j} K\left(x_{j}, x_{i}\right)-y_{i}
\end{aligned}
$$

Case $1 \alpha_{i}=0 \quad\left(F_{i}-b\right) y_{i} \geq 0$

Case $20<\alpha_{i}<C \quad\left(F_{i}-b\right) y_{i}=0$

Case $3 \alpha_{i}=C \quad\left(F_{i}-b\right) y_{i} \leq 0$

And by further classification according to the possible combination of alpha and $y_{i}$. We can have:

$$
\begin{aligned}
& b \leq F_{i} \text { for } \forall i \in I_{0} \cup I_{1} \cup I_{2} \\
& b \geq F_{i} \text { for } \forall i \in I_{0} \cup I_{3} \cup I_{4}
\end{aligned}
$$

\section{Material and Methods}

The block diagram of the proposed method for ECG beat classification shown in Figure 4. This method is separated into three steps:

- Pre Processing

- ECG Preprocessing

- Classification by SVM 
Citation: Sumathi S (2018) An Approach for ECG Feature Extraction and Classification of Cardiac Abnormalities. Cardiovasc Pharm Open Access 6: 234. doi: 10.4172/2329-6607.1000234

\section{Pre-processing}

Clinical ECG recordings experience a few stages of filtering in an attempt to decrease the noise. Each commotion source dwells in a characteristic recurrence band. Destitute conductance between skin and cathode makes gradually changing possibilities which shows as standard meander in the ECG. The output function of this WT will be our filtered signal. $\Psi(\mathrm{t})$, given in the equation (1). The parameters of this filtering are the attenuation factor $f$, and the basic frequency $f$.

$$
\Psi(\mathrm{t})=\exp \left(\frac{t^{2}}{\beta^{2}}\right) \cos (2 \Pi \mathrm{ft})-\lambda
$$

$\beta$ - Attenuation factor, $\mathrm{f}$ - base frequency, $\Psi(\mathrm{t})$ - Wavelet Transform, $\lambda$ - DC factor eliminator

Our goal is to find those parameter values and contribute the most to a good QRS detection ratio. The value of these parameters will be chosen so that it assures all the significant parts of the sum defined in Equation (1), but it eliminates all additional calculations for the attenuated part of the signal, that hardly influences the result. Elimination of this low and high frequency components from the signal using the filters is the concrete effect of the wavelet filtering shown in Figures 5-7.

In this article, the Gaussian kemel is used as the kernel function. Classification consists of two steps: learning and testing. Our classifier is a learning machine of the supervised type and Multicategory SVM (MC-SVM). Firstly, all ECG segments that contain a special type heartbeat are mapped into feature space using wavelet and AR model which have been explained above. In the learning phase, SVM receives some patterns as input. These patterns are heartbeats represented by $\mathrm{m}$ feature parameters that can be seen as points in rn-dimensional space. Then the machine becomes able to find the labels of new vectors by comparing them with those used in the learning phase.

\section{Results and Discussion}

In this article, the Gaussian kemel is utilized as the research work. Classification comprises of two steps: learning and testing. Our classifier is a learning machine of the administered sort and Multicategory SVM (MC-SVM). Firstly, all ECG fragments that contain a extraordinary sort pulse are mapped into highlight space utilizing wavelet and AR show which have been clarified over. In the learning stage, SVM gets a few designs as input. These designs are heartbeats spoken to by $\mathrm{m}$

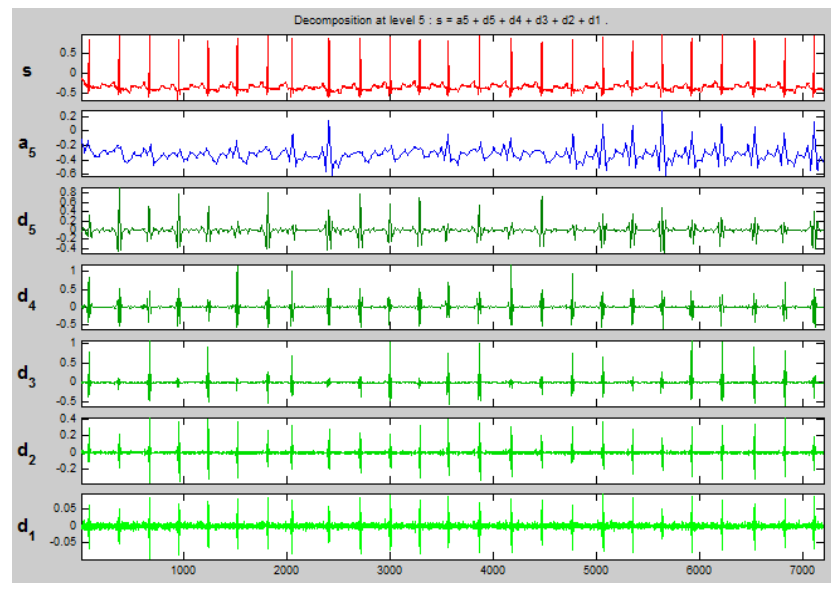

Figure 5: Decomposition of symlet Wavelet-original signal.
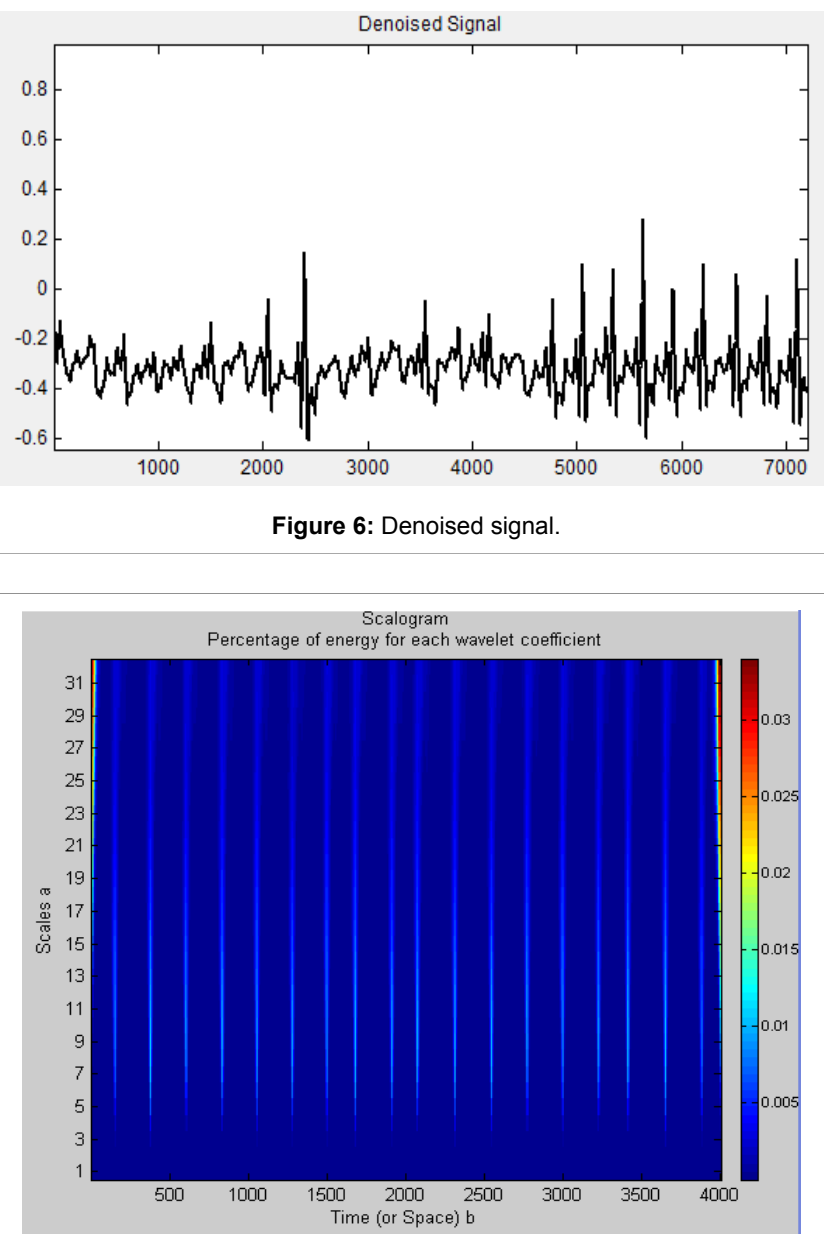

Figure 7: Scalogram from a clinical ECG recording using Symlet Wavelet.

\begin{tabular}{|c|c|c|c|c|}
\hline $\begin{array}{c}\text { Type of } \\
\text { Arrhythmia }\end{array}$ & $\begin{array}{c}\text { Training } \\
\text { Data }\end{array}$ & $\begin{array}{c}\text { Testing } \\
\text { Data }\end{array}$ & $\begin{array}{c}\text { Mis } \\
\text { Classification }\end{array}$ & $\begin{array}{c}\text { Classification } \\
\text { Accuracy (\%) }\end{array}$ \\
\hline VT & 75 & 30 & 1 & 94.33 \\
\hline VF & 95 & 40 & 1 & 97.00 \\
\hline VFLU & 85 & 35 & 1 & 94.11 \\
\hline $\begin{array}{c}\text { Myocardial } \\
\text { infarction }\end{array}$ & 95 & 40 & 1 & 97.00 \\
\hline $\begin{array}{c}\text { Myocardial } \\
\text { hypertrophy }\end{array}$ & 85 & 35 & 1 & 94.11 \\
\hline Total & 430 & 185 & 7 & 98.24 \\
\hline \multicolumn{4}{|c|}{ Table 1: Measurements based on ECG signals. } \\
\hline
\end{tabular}

highlight parameters that can be seen as focuses in rn-dimensional space. At that point the machine gets to be able to discover the names of modern vectors by comparing them with those utilized in the learning phase (Table 1).

The objective of this study is to model single-lead ECG signals for extricating classifiable highlights in arrange to improve the classification comes about utilizing wavelet and AR demonstrating. The wavelet change investigation gives strong highlights in nearness of foundation ceaseless commotion. Prevailing and critical highlights in the ECG information are extricated to supply strong data recovery for classification. AR coefficients are too be utilized to extricate the include of ECG information. Since of the diminished measurements of 
Citation: Sumathi S (2018) An Approach for ECG Feature Extraction and Classification of Cardiac Abnormalities. Cardiovasc Pharm Open Access 6: 234. doi: 10.4172/2329-6607.1000234

include vectors, the classification can be done rapidly. The test comes about appear using wavelet show together can result in tall precision of classification.

\section{References}

1. Chen (2000) A two stage discrimination of cardiac arrhythmias using a tota least squares-based prony modeling algorithm. IEEE Trans on BME 47: 13171327.

2. Giiler I, Ubeyli ED (2005) ECG beat classifier designed by combined neural network model. Rattern Recognition 38: 199-298.

3. Kikawa Y, Oguri K (2004) A Study for Excluding Incorrect Detections of Holter ECG Data Using SVM. ICONIP 3316: 1223-1228.

4. Osowski S, Hoai LT, Markiewicz T. Support Vector Machine-Based Expert System for Reliable Heartbeat Recognition. IEEE Transactions On Biomedical Engineering p: 51.

5. http://www.physionet.org
6. Luo ST, Nguyen Q, Afonso VX, Tompkins WJ (1999) ECG Beat Detection Using Filter Banks. IEEE Trans Biomed Eng 46: 192-202.

7. Hoai LT, Osowski S, Markiewicz T (2004) Support Vector Machine-Based Expert System for Reliable Heartbeat Recognition. IEEE Trans Biomed Eng 51: 582-589.

8. Robertson CE, Sterz F, Clegg GR, Watson JN, Holzer M, et al. (2000) Addison Evaluating Arrhythmias in ECG Signals Using Wavelet Transforms. IEEE Trans Biomed Eng PP: 104-109.

9. Addison S (2000) Evaluating Arrhythmias in ECG Signals Using Wavelet Transforms. IEEE Trans Biomed Eng PP: 104-109.

10. Ganapathiraju, Hamaker JH, Picone J (2004) Applications of support vector machines to speech recognition. IEEE Trans on Signal Processing 52: 2348-2355.

11. Daubechies (1990) The Wavelet Transform, Time-Frequency Localization and Signal Analysis. IEEE Trans Inform Theory PP: 961-1005.

12. Sumathi S, Sanavullah MY (2009) Comparative Study of QRS Complex Detection in ECG Based on Discrete Wavelet Transform. Int J Recent Trends in Engineering 2: 273-277. 